[7] J. Abadie, "Application of the GRG algorithm to optimal control problems," in Integer and Nonlinear Programming, J. Abadie, Ed. Amsterdam, The Netherlands: North-Holland Publ., 1970.

[8] H. J." Kelley, "A transformation approach to singular subares in optimal trajectory and control problems," $S Y A M J$. Contr., vol. 2, pp. $234-240,1964$.

[9] H.J. Kelley and W. F. Denham, "Modeling and adjoints for continuous systems," J. Optimiz. Theory Appl., Apr. 1969.

[10] W. Orchard-Hayes, Advanced Linear Programming Computing Techniques. New York: McGraw-Hill, 1968.

[11] R. E. Davis, "Computational procedures for optimal scheduling of hydroelectric power generation," Systems Control, Inc., Intern. Memo., July 1970.

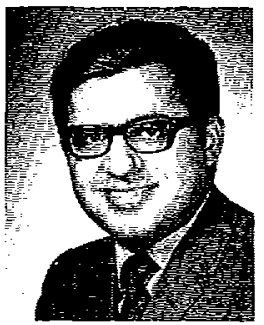

Raman K. Mehra (S'67-M'68) was born in Lahore, Pakistan, on February 10, 1943. He received the B.S. degree in electrical engineering from Punjab Engineering College, Chandigarh, India, in 1964, and the M.S. and $\mathrm{Ph} . \mathrm{D}$. degrees in engineering from $\mathrm{Har}-$ vard University, Cambridge, Mass., in 1965 and 1968 , respectively.

During the summer of 1965 he worked at Bell Telephone Laboratories, Inc., Andover, Mass. From 1966 to 1967 he was a Research Assistant at Harvard Lniversity. From September 1967 to September 1969 he was employed by the Analytic Sciences Corporation,
Reading, Mass., where he applied modern estimation and control theory to problems in inertial navigation. Since October 1969 he has been a Senior Research Engineer at Systems Control, Inc., Palo Alto, Calif. His interests lie in the areas of trajectory optimization; linear, nonlinear, and adaptive filtering; smasthing; system identification; and stochastic control. He is coauthor of the forthcoming book Applied Estimation Techniques (Academic Press, 1972).

Dr. Mehra is the recipient of the 1971 Donald P. Eckman Award for outstanding contributions to automatic control.

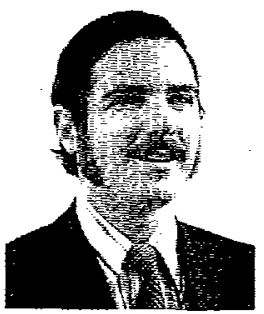

Ronald E. Davis was born in Lawton, Okla., on September 24, 1945. He received the B.A. degree in applied mathematics from Harvard University, Cambridge, Mass., and the M.S. degree in mathematics from Stanford University, Stanford, Calif., in 1967 and 1969 , respectively.

Since 1969 he has been working on optimization methods at Systems Control, Inc., Palo Alto, Calif. Implementable algorithms for mixed-integer large-scale nonlinear and stochastic dynamic programming problems have been developed in conjunction with work on specialization of nonlinear programming methods for optimal control problems. His main research interests are the identification, optimization, and control of socio-economic systems with multiple objectives.

\title{
The Maximally Achievable Accuracy of Linear Optimal Regulators and Linear Optimal Filters
}

\author{
HUIBERT KWAKERNAAK, MEMBER, IEEE, AND RAPHAEL SIVAN
}

\begin{abstract}
A linear system with a quadratic cost function, which is a weighted sum of the integral square regulation error and the integral square input, is considered. What happens to the integral square regulation error as the relative weight of the integral square input reduces to zero is investigated. In other words, what is the maximum accuracy one can achieve when there are no limitations on the input? It turns out that the necessary and sufficient condition for reducing the regulation error to zero is that 1) the number of inputs be at least as large as the number of controlled variables, and 2) the system possess no right-half plane zeros. These results are also "dualized" to the optimal filtering problem.
\end{abstract}

\section{INTRODUCTION}

$\mathbf{I}^{\mathrm{N}}$ designing a control system, it is usually necessary to make a tradeoff between achieving better performance and using smaller actuating forces. Namely, if one is willing to use higher power (or amplitude) levels at the input of a

Manuscript received January 7, 1971; revised May 11, 1971, and September 3, 1971. Paper recommended by P. Dorato, past Chairman of the IEEE G-AC Optimal Systems Committee.

H. Kwakernaak is with the Department of Applied Mathematics, Twente University of Technology, Enschede, The Netherlands.

R. Sivan is with the Department of Electrical Engineering, Technion-Israel Institute of Technology, Haifa, Israel. plant, one can usually achieve smaller deviations of the controlled variable from its desired trajectory. The following question thus comes up. Assuming that the input power is not limited, can one achieve perfect performance, or is there a lower bound on the performance that cannot be surpassed? In this paper this question will be answered; in fact, systems will be classified into the two following groups.

1) Systems with unlimited accuracy are those for which the performance index can be reduced to zero if the amplitudes of the input are allowed to increase indefinitely.

2) Systems with limited accuracy are those for which the performance index cannot be reduced beyond a certain value, even if the input amplitudes are allowed to increase indefinitely.

Our main result is that systems for which the number of inputs are larger than or equal to the number of controlled variables, and which possess the property that the transfer matrix of the system has no zeros in the right-half complex plane, comprise the class of systems with unlimited accuracy. This result agrees with the well-known fact that 
systems with right-half plane zeros have certain defciencies that make them less easy to control [1].

We shall consider the linear time-invariant quadratic cost optimal regulator problem. The system equations are

$$
\begin{gathered}
\dot{x}(t)=A x(t)+B u(t), \quad x\left(t_{0}\right)=x_{0} \\
z(t)=D x(t)
\end{gathered}
$$

where $x(t)$, the state, is an $n$-dimensional vector; $u(t)$, the input, is a $k$-dimensional vector; $z(t)$, the controlled variable, is a $p$-dimensional vector; and $x_{0}$ is the initial state at time $t_{0}$.

Let $C$ be the performance criterion to be minimized,

$$
C=\int_{t_{0}}^{\infty}\left[z^{T}(t) R_{3} z(t)+u^{T}(t) R_{2} u(t)\right] d t
$$

where $R_{3}$ and $R_{2}$ are symmetric positive-definite matrices. It will be convenient to rewrite the criterion as follows

$$
C=\int_{t_{0}}^{\infty}\left[C_{e}(t)+\rho C_{u}(t)\right] d t
$$

where $C_{e}(t)$,

$$
C_{e}(t)=z^{T}(t) R_{3} z(t),
$$

is the square regulation error; $C_{u}(t)$,

$$
C_{u}(t)=u^{T}(t) N u(t),
$$

is the square input; and $\rho$ is a positive scalar which determines the relative weight of $C_{e}(t)$ and $C_{u}(t)$. It follows that

$$
R_{2}=\rho N
$$

with $N$ a symmetric positive-definite matrix.

A typical design procedure for a regulator would be as follows. First one solves the optimization problem for a given set of values of $R_{3}, N$ and $\rho$ [2]. The next step in the design is to evaluate separately the integral square regulation error

$$
\int_{t_{0}}^{\infty} C_{e}(t) d t
$$

and the integral square input

$$
\int_{t_{0}}^{\infty} C_{u}(t) d t
$$

for the optimally designed system. If it turns out that the integral square regulation error is too large, we decrease $\rho$ and again solve the optimization problem. This will result in a lower integral square regulation error at the expense of a larger integral square input.

In this paper we shall investigate the limit

$$
\lim _{\rho \downarrow 0} \int_{t_{0}}^{\infty} C_{e}(t) d t
$$

and classify systems of unlimited accuracy, for which

$$
\lim _{\rho \downarrow 0} \int_{t_{0}}^{\infty} C_{e}(t) d t=0, \quad \text { for all } x_{0},
$$

and systems of limited accuracy, for which

$$
\lim _{\rho \downarrow 0} \int_{t_{0}}^{\infty} C_{e}(t) d t>0, \quad \text { for some } x_{0}
$$

In the last section, we shall dualize the notions and results of this paper to the optimal filtering problem where we shall classify filters as filters with unlimited accuracy and filters with limited accuracy.

The results of this paper are related to those of Friedland [3] and Kwakernaak [4], where the effect of taking the limit $\rho \downarrow 0$ on the form of the optimal control law is discussed.

\section{RESULTS AND INTERPRETATION}

Before stating the main results of this paper, we introduce the notions of numerator polynomial and zeros of a multi-input multi-output linear time-invariant system for systems where the number of inputs are the same as the number of controlled variables.

Definition [5], [6]: Consider the system (1), (2) for the case that $k=p$, and denote by

$$
H(s)=D(s I-A)^{-1} B,
$$

the $k \times k$ transfer matrix of this system. Let $\phi(s)$ denote the characteristic polynomial of $A$ and write

$$
\operatorname{det}[H(s)]=\frac{\psi(s)}{\phi(s)}
$$

where $\psi(s)$ is a polynomial in $s$ of degree $n-k$ or less. Then $\psi(s)$ is called the numerator polynomial of the system and its roots are called the zeros of the system.

Note that in the special case where the system is singleinput single-output, $\psi(s)$ is just the numerator of the transfer function, and its roots are commonly referred to as the zeros of the transfer function, provided no cancellations occur.

We are now ready to state our main result.

\section{TheOREM}

Consider the time-invariant stabilizable and detectable linear system

$$
\begin{aligned}
& \dot{x}(t)=A x(t)+B u(t) \\
& z(t)=D x(t)
\end{aligned}
$$

with $\operatorname{dim}(u) \leq \operatorname{dim}(x), \operatorname{dim}(z) \leq \operatorname{dim}(x)$, and where $B$ and $D$ are assumed to have full rank. Consider also the criterion

$$
\int_{t_{0}}^{\infty}\left[z^{T}(t) R_{3} z(t)+u^{T}(t) R_{2} u(t)\right] d t
$$

where $R_{3}$ and $R_{2}$ are positive-definite symmetric matrices. Let

$$
R_{2}=\rho N
$$

with $N$ positive definite and $\rho$ a positive scalar, and let $\bar{P}$ be the steady-state solution of the Riccati equation 
$-\dot{P}(t)=D^{T} R_{3} D-P(t) B R_{2}{ }^{-1} B^{T} P(t)+A^{T} P(t)+P(t) A$, $P\left(t_{1}\right)=0$.

Then the following facts hold.

Fact 1: The limit

$$
\lim _{\rho \downarrow 0} \bar{P}=P_{0}
$$

exists. lator,

Fact 2: For the closed-loop steady-state optimal regu-

$$
\lim _{\rho \downarrow 0} \int_{t_{0}}^{\infty} z^{T}(t) R_{3} z(t) d t=x^{T}\left(t_{0}\right) P_{0} x\left(t_{0}\right) .
$$

Fact 3: If $\operatorname{dim}(z)>\operatorname{dim}(u)$, then $P_{0} \neq 0$.

Fact 4: If $\operatorname{dim}(z)=\operatorname{dim}(u)$ and the numerator polynomial $\psi(s)$ of the open-loop transfer matrix $H(s)=$ $D(s I-A)^{-1} B$ is nonzero, $P_{0}=0$ if and only if all the zeros of the numerator polynomial $\psi(s)$ have nonpositive real parts.

Fact 5: If $\operatorname{dim}(z)<\operatorname{dim}(u)$, then $P_{0}=0$ if there exists a rectangular matrix $M$ such that the numerator polynomial $\psi^{\prime}(s)$ of the square transfer matrix $D(s I-A)^{-1} B M$ is nonzero and has zeros with nonpositive real parts only.

It is recalled that the system (15) is stabilizable if there exists a constant matrix $F$ such that the matrix $A-B F$ has all its characteristic values in the left-half complex plane [7]. Similarly, the system (15) is detectable if there exists a constant matrix $K$ such that the matrix $A-K D$ has all its characteristic values in the left-half complex plane.

A discussion of the significance of the various parts of the theorem now follows. Fact 1 states that as we let the weighting coefficient of the input $\rho$ decrease the minimal value of the criterion,

$$
\min \int_{t_{0}}^{\infty}\left[z^{T}(t) R_{3} z(t)+\rho u^{T}(t) N u(t)\right] d t=x^{T}\left(t_{0}\right) \bar{P} x\left(t_{0}\right)
$$

approaches the limit $x^{T}\left(t_{0}\right) P_{0} x\left(t_{0}\right)$ as $\rho \downarrow 0$.

Next, using the notation (5), (6), it follows from Fact 2 of the theorem that

$$
\lim _{\rho \downarrow 0}\left\{\rho \int_{t_{0}}^{\infty} C_{u}(t) d t\right\}=0
$$

so that in the limit as $\rho \downarrow 0$ the integral square regulating error fully accounts for the cost $C(4)$.

Facts 3,4 , and 5 of the theorem are concerned with the conditions under which $P_{0}=0$. It is under these conditions that ultimately perfect regulation is achieved since

$$
\lim _{\rho \downarrow 0} \int_{t_{0}}^{\infty} C_{e}(t) d t=0, \quad \text { for all } x_{0}
$$

Fact 3 of the theorem states that, if the dimension of the controlled variable is greater than that of the input, perfect, regulation is impossible. This is very reasonable since in this case the number of degrees of freedom to control the system is too small. In order to determine the maximal accuracy that may be achieved, $P_{0}$ must be computed. In the section on the filtering problem we shall give a hint how $P_{0}$ may be found.

In Fact 4 the case is considered where the number of degrees of freedom are sufficient, i.e., the input and the controlled variable have the same dimensions. Here the maximally achievable accuracy is dependent upon the properties of the open-loop system transfer matrix $H(s)$. Perfect regulation is only possible provided the numerator polynomial $\psi(s)$ of the transfer matrix has no right-half plane zeros (assuming that $\psi(s)$ is not identical to zero). This may be made intuitively plausible by considering the limiting situation when $\rho=0$. Let $t_{0}=0^{-}$and suppose that at time $0^{-}$the system is in the initial state $x_{0}$. Then in terms of Laplace transforms the response of the controlled variable may be expressed as

$$
Z(s)=H(s) U(s)+D(s I-A)^{-1} x_{0}
$$

where $Z(s)$ and $U(s)$ are the Laplace transforms of $z$ and $u$, respectively. The time function $z(t)$ can be made identical to zero for $t \geq 0$ by choosing

$$
U(s)=-H^{-1}(s) D(s I-A)^{-1} x_{0} .
$$

The input $u(t)$ is actually made up of $\delta$-functions and derivatives of $\delta$-functions at time $t=0$. These $\delta$-functions instantaneously transfer the state $x_{0}$ at time $0^{-}$to a state $x(0)$ at time 0 , which has the property that $z(0)=D x(0)$ $=0$ and that $z(t)$ can be maintained at 0 for $0 \leq t<\infty$ [8]. Note that in general the state $x(t)$ will undergo a $\delta$-function and derivative of $\delta$-function type of trajectory but $z(t)$, as can be seen by inserting (25) into (24), will move from $z\left(0^{-}\right)=D x_{0}$ to $z(0)=0$ directly, with no infinite excursions.

This input (25) will lead to a stable behavior of the input only if the inverse transfer matrix $H^{-1}(s)$ is stable, namely, if the numerator polynomial $\psi(s)$ of $H(s)$ has no right-half plane zeros.

The reason that the input (25) cannot be used in the case where $H^{-1}(s)$ has unstable poles is that the input $u(t)$ as given by (25) will drive $z(t)$ to zero without $z(t)$ having any $\delta$-functions, and this $u(t)$ will also maintain $z(t)$ at zero for $t \geq 0$, but $u(t)$ will have to grow indefinitely since (25) has right-half plane zeros [9]. By our problem formulation and also by considerations of practical applicability, such inputs are ruled out so that in this case (25) is not the limiting input as $\rho \downarrow 0$, and in fact, costless regulation cannot be achieved.

However, note that if $R_{2}=0$ from the outset [10], we do not rule out an indefinitely growing input and $u(t)$ given by $(25)$ is the solution irrespective of the location of the zeros of the system, as long as $H^{-1}(s)$ exists. Such a problem formulation, however, has little practical significance.

Note that for single-input single-output systems the condition that all zeros be in the left-half plane amounts to the requirement that the system transfer function have no right-half plane zeros. It is well known to control engineers 
[1] that systems with right-half plane zeros possess inherent limitations that cannot be overcome by "tightening the feedback loop."

The results of this part of the theorem agree with a related fact that has been discussed elsewhere [11], and which concerns the asymptotic behavior of the closed-loop regulator poles, i.e., the characteristic values of the matrix $A-B R_{2}^{-1} B^{T} \bar{P}$. As it turns out, those closed-loop poles that do not go to infinity as $\rho \downarrow 0$ approach the numbers $\hat{\nu}_{i}, i=1,2, \cdots, m$, which are related to the zeros $\nu_{i}, i=$ $1,2, \cdots, m$, of the numerator polynomial $\psi(s)$ as follows:

$$
\hat{\nu}_{i}=\left\{\begin{aligned}
\nu_{i} & \text { if } \operatorname{Re}\left(\nu_{i}\right) \leq 0 \\
-\nu_{i} & \text { if } \operatorname{Re}\left(\nu_{i}\right)>0
\end{aligned}\right\} .
$$

(This is a generalization of a fact that is well known in the single-input single-output case [12].) Thus, if the system has no right-half plane zeros, in the limit as $\rho \downarrow 0$, exactly $m$ closed-loop poles coincide with the system zeros $\nu_{i}, i=$ $1,2, \cdots, m$. Apparently, these nearby poles are "cancelled" by the system zeros, and the response of the controlled variable is completely determined by the far-away closedloop poles, resulting in an arbitrarily fast response. On the other hand, if the system possesses one or more right-half plane zeros, cancellation does not take place and the speed of response, and hence the accuracy of the regulator, is limited.

Finally, in Fact 5 of the theorem we state a sufficient condition for $P_{0}$ to be zero for the case where the number of inputs are larger than the number of outputs. The idea is to replace the input $u$ with an input $u^{\prime}$,

$$
u^{\prime}(t)=M u(t)
$$

where $u^{\prime}$ is a linear transformation of $u$, the dimension of which is the same as the dimension of the output. ${ }^{1}$

The results of the theorem pertain to the deterministic linear optimal regulator problem. They are also of interest for related problems such as the stochastic linear optimal regulator problem and the linear optimal tracking problem, since they are closely associated with the deterministic regulator problem.

\section{Proof of the Theorem}

First we consider Fact 1. $\bar{P}$ is defined by

$$
\begin{aligned}
x^{T}\left(t_{0}\right) \bar{P} x\left(t_{0}\right)=\min _{\left\{u(t), t \geq t_{0}\right\}}\left\{\int _ { t _ { 0 } } ^ { \infty } \left[z^{T}(t) R_{3} z(t)\right.\right. \\
\\
\left.\left.\quad+\rho u^{T}(t) N u(t)\right] d t\right\} .
\end{aligned}
$$

Clearly as a function of $\rho$ this expression has zero as a lower bound. Moreover, this expression is monotonically nonincreasing with decreasing $\rho$. This may be seen as follows. Suppose that the minimization is carried out for a particular value of $\rho$. Then if $\rho$ is decreased and the same

\footnotetext{
1 Moore and Silverman [17] have pointed out to the authors that it may be shown by a counterexample that the condition in Fact 5 of
} the theorem is sufficient but not necessary. solution is maintained, the expression in braces decreases. If the minimization is repeated for this smaller value of $\rho$, only an even smaller value can result. Thus (28) is nonincreasing with decreasing $\rho$; because it also has a lower bound it must have a limit as $\rho \downarrow 0$. Since this limit exists for all $x\left(t_{0}\right), \bar{P}$ must have a limit that we denote as $P_{0}$.

In the following, let $u_{\rho}(t), t \geq t_{0}$, denote the input that is optimal for a given initial state (which is fixed) and a given value of $\rho$. Similarly, $z_{\rho}(t), t \geq t_{0}$, denotes the resulting behavior of the controlled variable. Fact 2 of the theorem is now proved as follows. The integral square regulation error

$$
\int_{t_{0}}^{\infty} z_{\rho}^{T}(t) R_{3} z_{\rho}(t) d t
$$

has zero as a lower bound. Moreover, it is nonincreasing with decreasing $\rho$ since a smaller value of $\rho$ results in a larger integral square input and thus in a smaller integral square regulation error. Hence, (29) has a limit for $\rho \downarrow 0$. Since for all $\rho, \rho>0$,

$$
\begin{aligned}
\int_{t_{0}}^{\infty}\left[z_{\rho}^{T}(t) R_{3} z_{\rho}(t)+\rho u_{\rho}^{T}(t) N u_{\rho}(t)\right] d t & \\
& \geq \int_{t_{0}}^{\infty} z_{\rho}^{T}(t) R_{3} z_{\rho}(t) d t
\end{aligned}
$$

we must have

$$
\lim _{\rho \downarrow 0} \int_{t_{0}}^{\infty} z_{\rho}{ }^{T}(t) R_{3} z_{p}(t) d t \leq x^{T}\left(t_{0}\right) P_{0} x\left(t_{0}\right) .
$$

Suppose that this is a strict inequality; then there must exist an $\epsilon>0$ such that

$$
\lim _{\rho \downarrow 0} \int_{t_{0}}^{\infty} z_{\rho}^{T}(t) R_{3} z_{\rho}(t) d t=x^{T}\left(t_{0}\right) P_{0} x\left(t_{0}\right)-\epsilon .
$$

Then we can always find a value of $\rho$, say $\rho_{0}$, such that

$$
\int_{t_{0}}^{\infty} z_{\rho_{0}}{ }^{T}(t) R_{3} z_{\rho_{0}}(t) d t=x^{T}\left(t_{0}\right) P_{0} x\left(t_{0}\right)-\frac{\epsilon}{2}
$$

Since

$$
\int_{t_{0}}^{\infty} u_{\rho_{0}}{ }^{T}(t) N u_{\rho 0}(t) d t
$$

is finite, we can always select a positive $\rho_{1}$ such that

$$
\rho_{1} \int_{t_{0}}^{\infty} u_{\rho 0}^{T}(t) N u_{\rho 0}(t) d t<\frac{\epsilon}{4}
$$

Then we have

$$
\begin{aligned}
\int_{t_{0}}^{\infty}\left[z_{\rho 0}{ }^{T}(t)\right. & \left.R_{3} z_{\rho 0}(t)+\rho_{1} u_{\rho 0}{ }^{T}(t) N u_{\rho 0}(t)\right] d t \\
& <x^{T}\left(t_{0}\right) P_{0} x\left(t_{0}\right)-\frac{\epsilon}{2}+\frac{\epsilon}{4}<x^{T}\left(t_{0}\right) P_{0} x\left(t_{0}\right)
\end{aligned}
$$

This is a contradiction since for no positive value of $\rho$ there exists an input that makes

$$
\int_{t_{0}}^{\infty}\left[z^{T}(t) R_{3} z(t)+\rho u^{T}(t) R_{2} u(t)\right] d t
$$


less than $x^{T}\left(t_{0}\right) P_{0} x\left(t_{0}\right)$. Hence the inequality sign in (31) cannot hold and Fact 2 of the theorem is true.

To prove Facts 3, 4, and 5, we first consider the algebraic Riccati equation

$$
0=D^{T} R_{3} D-\frac{1}{\rho} \bar{P} B N^{-1} B^{T} \bar{P}+\breve{P} A+A^{T} \tilde{P}
$$

We shall investigate the hypothesis that as $\rho \downarrow 0, \vec{P}$ approaches $P_{0}=\mathbf{0}$. Since the first term of (38) is independent of $\rho$ and is finite and, according to the hypothesis, the last two terms approach zero, we have

$$
\lim _{\rho t_{0}} \frac{\bar{P}}{\sqrt{\rho}} B N^{-1} B^{T} \frac{\bar{P}}{\sqrt{\rho}}=D^{T} R_{3} D \text {. }
$$

Since $N$ is nonsingular, the limit

$$
L=\lim _{\rho \downarrow 0} B^{T} \frac{\bar{P}}{\sqrt{\rho}}
$$

must exist. For $L$ we have the equality

$$
L^{T} N^{-1} L=D^{T} R_{3} D .
$$

We shall investigate under which conditions this equation has a solution for $L$. We first state the following fact from matrix theory.

Lemma: Consider the matrix equation

$$
X^{T} X=C
$$

where $X$ is an unknown $p \times q$ matrix with $p \leq q$ and $C$ a known $q \times q$ nonnegative-definite symmetric matrix. This equation has a solution if and only if

$$
\operatorname{rank}(C) \leq p
$$

If this condition is satisfied the general solution of (42) may be expressed as

$$
X=U Y
$$

where the $p \times q$ matrix $Y$ is any solution of (42) and $U$ is an arbitrary $p \times p$ unitary matrix.

We recall that $U$ is a unitary matrix if

$$
U^{T} U=I \text {. }
$$

This lemma is easily proved by first reducing $C$ to diagonal form and then to the unit matrix. Let us apply this result to (41), which we first rewrite as

$$
\left(N^{-1 / 2} L\right)^{T}\left(N^{-1 / 2} L\right)=\left(R_{3}^{1 / 2} D\right)^{T}\left(R_{3}{ }^{1 / 2} D\right) .
$$

Here if $M$ is a nonnegative-definite symmetric matrix, $M^{1 / 2}$ is the unique nonnegative-definite matrix that satisfies $M^{1 / 2} M^{1 / 2}=M$; furthermore, $M^{-1 / 2}=\left(M^{-1}\right)^{1 / 2}=$ $\left(M^{1 / 2}\right)^{-1}$. Now

$$
N^{-1 / 2} L=\lim _{\rho \rightarrow 0} N^{-1 / 2} B^{T} \frac{\bar{P}}{\sqrt{\rho}}
$$

has the dimensions $k \times n$, where $k$ is the dimension of the system and $n$ that of the state $x$. We see therefore from the lemma that (46) has a solution $L$ if and only if

$$
\operatorname{rank}\left(D^{T} R_{3} D\right) \leq k
$$

or equivalently, if and only if

$$
\operatorname{rank}\left(R_{3}{ }^{1 / 2} D\right) \leq k .
$$

But since $R_{3}{ }^{1 / 2}$ is square and nonsingular, rank $\left(R_{3}{ }^{1 / 2} D\right)=$ rank $(D)$, and consequently, (46) has a solution if and only if

$$
\operatorname{rank}(D) \leq k \text {. }
$$

Since by assumption $D$ has full rank, it follows that (46) has a solution if and only if

$$
\operatorname{dim}(z) \leq \operatorname{dim}(u),
$$

i.e., the number of components of the controlled variable must not exceed the number of components of the input variable. Now, if the condition (51) is violated, (46) does not have a solution $L$. This means that (39) cannot be true, which implies that the hypothesis that $P_{0}=0$ is false. Thus we have shown that if we attempt to regulate a controlled variable of higher dimension than the input, it is never possible to achieve an arbitrarily small value of the optimization criterion. This proves Fact 3 of the theorem.

We continue the analysis under the assumption that

$$
\operatorname{dim}(z)=\operatorname{dim}(u) .
$$

Then we can write for the solution of (46)

$$
N^{-1 / 2} L=U R_{3}^{1 / 2} D
$$

where $U$ is an arbitrary unitary matrix. To see whether this expression is still consistent with the hypothesis $P_{0}=\mathbf{0}$, let us consider the closed-loop characteristic polynomial as $\rho \downarrow 0$. We introduce the notation

$$
\bar{F}=\frac{1}{\rho} N^{-1} B^{T} \bar{P}
$$

and write

$$
\begin{aligned}
\operatorname{det} & (s I-A+B \bar{F}) \\
= & \operatorname{det}(s I-A) \operatorname{det}\left[I+B \bar{F}(s I-A)^{-1}\right] \\
= & \operatorname{det}(s I-A) \operatorname{det}\left[I+\bar{F}(s I-A)^{-1} B\right] \\
= & \operatorname{det}(s I-A) \operatorname{det}\left[I+\frac{1}{\rho} N^{-1} B^{T} \bar{P}(s I-A)^{-1} B\right] \\
= & \operatorname{det}(s I-A) \\
& \cdot \operatorname{det}\left[I+\frac{1}{\sqrt{\rho}} N^{-1} B^{T} \frac{\bar{P}}{\sqrt{\rho}}(s I-A)^{-1} B\right] .
\end{aligned}
$$

It is seen from (55) that those closed-loop characteristic values that stay finite as $\rho \downarrow 0$ approach the roots of

$$
\operatorname{det}(s I-A) \operatorname{det}\left[N^{-1} L(s I-A)^{-1} B\right] \text {, }
$$

since under our hypothesis

$$
\lim _{\rho \downarrow 0} B^{T} \frac{\bar{P}}{\sqrt{\rho}}=L
$$


With (53) it follows that the nearby closed-loop characteristic values are the zeros of

$$
\begin{array}{r}
\operatorname{det}(s I-A) \operatorname{det}\left[N^{-1 / 2} U R_{3}{ }^{1 / 2} D(s I-A)^{-1} B\right] \\
=\operatorname{det}\left(N^{-1 / 2} U R_{3}{ }^{1 / 2}\right) \psi(s)
\end{array}
$$

where $\psi(s)$ is the numerator polynomial of the transfer matrix $H(s)$, i.e.,

$$
\operatorname{det}[H(s)]=\frac{\psi(s)}{\phi(s)} .
$$

We know that the closed-loop characteristic values are in the left-half complex plane since the closed-loop system is asymptotically stable [7]. Our present conclusion is that the nearby closed-loop poles are the zeros of the transfer matrix $H(s)$. This conclusion can only be correct if $H(s)$ has left-half plane zeros only. If $H(s)$ possesses one or more right-half plane zeros, our conclusion is wrong and the hypothesis that $P_{0}=0$ is false. Thus we have shown that if $\operatorname{dim}(u)=\operatorname{dim}(z)$ but $H(s)$ has right-half plane zeros, $P_{0} \neq 0$. This proves one direction of Fact 4 of the theorem.

We have now shown that $P_{0} \neq 0$ in the following cases: 1) $\operatorname{dim}(u)<\operatorname{dim}(z)$, and 2) $\operatorname{dim}(u)=\operatorname{dim}(z)$ and the numerator of the open-loop transfer matrix $D(s I-A)^{-1} B$ is nonzero and has one or more right-half plane zeros.

We shall now constructively show when $P_{0}=0$. We let $\operatorname{dim}(u)=\operatorname{dim}(z)$ and assume that $H(s)=D(s I-A)^{-1} B$ has left-half plane zeros only, including the imaginary axis. If $\operatorname{dim}(u)>\operatorname{dim}(z)$, we assume that there exists a matrix $M$ such that the numerator of the square transfer matrix $D(s I-A)^{-1} B M$ is nonzero and has left-half plane zeros only, including the imaginary axis. Since the latter case is equivalent to replacing the input $u(t)$ with an input $u^{\prime}(t)$ such that

$$
u(t)=M u^{\prime}(t),
$$

we need only consider the case where $\operatorname{dim}(z)=\operatorname{dim}(u)$.

Furthermore, we assume that the open-loop system is asymptotically stable, i.e., the matrix $A$ has all its characteristic values in the left-half complex plane (without the imaginary axis). If this is not the case, due to the assumption of stabilizability it is always possible to connect a feedback law

$$
u(t)=-F x(t)+u^{\prime}(t)
$$

that stabilizes the system. This feedback law leaves the numerator polynomial of the transfer matrix unchanged, (see [16, proposition 2]).

Consider now the response of the system

$$
\begin{aligned}
\dot{x}(t) & =A x(t)+B u(t) \\
z(t) & =D x(t)
\end{aligned}
$$

to an arbitrary initial state $x\left(0^{-}\right)=x_{0}$. Laplace transformation yields

$$
Z(s)=H(s) U(s)+D(s I-A)^{-1} x_{0} .
$$

Now suppose that we choose

$$
U(s)=-H^{-1}(s) D(s I-A)^{-1} x_{0} .
$$

Then (63) shows that we obtain a response $z(t) \equiv 0, t \geq 0$. The input characterized by (64), however, will usually not be physically realizable (it contains delta functions and derivatives of delta functions) since the expression (64) has terms in the numerator of a higher degree in $s$ than the denominator. We therefore consider the input with Laplace transform

$$
U_{\alpha}(s)=H^{-1}(s) K_{\alpha}(s) D(s I-A)^{-1} x_{0}
$$

where $K_{\alpha}(s)$ is of the form

$$
K_{\alpha}(s)=\frac{\alpha^{l}}{(s+\alpha)^{l}} I .
$$

Here the integer $l$ is so chosen that the degree of the denominator of (65) is higher than that of the numerator, and $\alpha$ is a positive real scalar. Now (66) represents the Laplace transform of a realizable input. In order to prove that the limit of the minimal cost is zero, i.e.,

$$
\lim _{\rho \downarrow 0} \min _{u} C=0 \text {, }
$$

we shall show that for every $\epsilon>0$ there exist an $\alpha^{*}$ and a $\rho^{*}\left(\alpha^{*}\right)$ so that $C(\alpha, \rho)<\epsilon$ for $\alpha=\alpha^{*}$ and $0<\rho<\rho^{*}\left(\alpha^{*}\right)$.

With (63) we find the response to the input $U_{\alpha}(s)$ to be

$$
\begin{aligned}
Z_{\alpha}(s) & =\left[I-K_{\alpha}(s)\right] D(s I-A)^{-1} x_{0} \\
& =\left[1-\frac{\alpha^{l}}{(s+\alpha)^{l}}\right] D(s I-A)^{-1} x_{0} .
\end{aligned}
$$

From Parseval's theorem it follows

$$
\begin{aligned}
\int_{0}^{\infty} z_{\alpha}{ }^{T}(t) R_{3} z_{\alpha}(t) d t= & \int_{-\infty}^{\infty} Z_{\alpha}^{T}(-j 2 \pi f) R_{3} Z_{\alpha}(j 2 \pi f) d f \\
= & \int_{-\infty}^{\infty}\left|1-\frac{\alpha^{l}}{(j \omega+\alpha)^{2}}\right|^{2} \\
& \cdot x_{0}^{T}\left(-j \omega I-A^{T}\right)^{-1} \\
& \cdot D^{T} R_{3} D(j \omega I-A)^{-1} x_{0} d f
\end{aligned}
$$

where $\omega=2 \pi f$. This equality is valid since by assumption $A$ has left-half plane characteristic values only and $\alpha$ is positive. It is not difficult to prove that this expression may be made arbitrarily small by making $\alpha$ large enough. Let us choose $\alpha^{*}$ so that for $\alpha=\alpha^{*}$ the first term in the criterion

$$
\int_{0}^{\infty}\left[z_{\alpha}^{T}(t) R_{3} z_{\alpha}(t)+u_{\alpha}^{T}(t) R_{2} u_{\alpha}(t)\right] d t
$$

is smaller than $\epsilon / 2$. With the input (65) and $\alpha=\alpha^{*}$ we can write, for the second term, 


$$
\begin{aligned}
\int_{0}^{\infty} u_{\alpha^{*}}{ }^{T}(t) R_{2} u_{\alpha^{*}}(t) d t= & \rho \int_{-\infty}^{\infty} x_{0}^{T}\left(-j \omega I-A^{T}\right)^{-1} \\
& \cdot K_{\alpha^{*}}(-j \omega)\left[H^{-1}(-j \omega)\right]^{T} \\
& \cdot N H^{-1}(j \omega) \\
& \cdot K_{\alpha^{*}}(j \omega)(j \omega I-A)^{-1} x_{0} d f
\end{aligned}
$$

where we have replaced $R_{2}$ with $\rho N$. This step is allowed since by assumption $A$ is stable, $K_{\alpha^{*}}(s)$ has left-half plane poles, and $H^{-1}(s)$ has left-half plane poles only by the assumption that the numerator of $H(s)$ has left-half plane zeros only. (If $H^{-1}(s)$ has poles on the imaginary axis, we can make a slight perturbation to bring them into the lefthalf plane; this does not essentially change the argument.) Since the integral on the right-hand side is finite, we can now choose $\rho^{*}$ so that the right-hand side of $(70)$ is less than $\epsilon / 2$. Thus we have proved that under the assumptions stated the criterion (21) may be made arbitrarily close to zero by making $\rho$ small. This shows that the minimum value of (21) approaches zero as $\rho \downarrow 0$ and, consequently, that $P_{0}=0$. This terminates the proof of Fact 4 of the theorem and also proves Fact 5.

\section{Filtering with Limited Accuracy and Filmering With UnLIMITEd ACCURACY}

We shall very briefly transfer the above results to the filter problem, which is dual to the regulator problem [13].

Consider the system

$$
\begin{aligned}
& \dot{x}(t)=A x(t)+B v_{1}(t) \\
& y(t)=D x(t)+v_{2}(t)
\end{aligned}
$$

where $v_{1}$ and $v_{2}$ are uncorrelated white-noise processes with intensities $V_{1}$ and $V_{2}$. It is assumed that both $V_{1}$ and $V_{2}$ are positive definite. It is well known [10] that using the optimal filter,

$$
\lim _{t \rightarrow \infty} E\left\{e^{T}(t) e(t)\right\}=\operatorname{tr}(\bar{Q})
$$

where $e$ is the estimation error and $\bar{Q}$ is the nonnegativedefinite solution of the equation

$$
A \bar{Q}+\bar{Q} A^{T}+B V_{1} B^{T}-\bar{Q} D^{T} V_{2}^{-1} D \bar{Q}=0 .
$$

Let us denote

$$
V_{2}=\rho N
$$

and investigate the limit

$$
\lim _{\rho \downarrow 0} \operatorname{tr}(\bar{Q}) \text {. }
$$

Namely, let us find out how far the estimation error can be reduced if we are willing to perfect the measuring equipment up to the point where it is practically noise free. In particular, let us find out whether the mean-square estimation error $\operatorname{tr}(\bar{Q})$ reduces to zero as the measurement noise decreases to zero. The following corollary states the conditions under which $\bar{Q}$ tends to the zero matrix.
Corollary: If $A, B$, and $D$ of a filtering problem are identical to the $A^{T}, D^{T}$, and $B^{T}$, respectively, of a regulation problem, then

$$
\lim _{\rho \downarrow 0} \bar{Q}
$$

equals the zero matrix if and only if $P_{0}$ of the regulation problem equals the zero matrix.

Thus, roughly, we can state that the estimation error can be reduced to zero by reducing the measurement noise to zero if and only if 1 ) the number of observed variables $[=\operatorname{dim}(y)]$ are at least as large as the number of disturbing variables $\left[=\operatorname{dim}\left(v_{1}\right)\right]$ and (2) when the number of observed variables equal the number of disturbing variables, the zeros of the square transfer matrix $D(s I-A)^{-1} B$ are all in the left-half complex plane.

We conclude this section by pointing out that the limit (77) may be computed by solving the singular optimal filtering problem [14] that results from setting $V_{2}=0$. Similarly, for the regulation problem the limit $P_{0}$ may be computed by determining the dual filtering problem [5] and solving the singular dual filtering problem that results by setting $R_{2}=0$. The papers of Butman [15] and Friedland [3] contain contributions to these problems.

\section{Conclusion}

This paper has established the connection between the maximally achievable accuracy and the minimally achievable estimation error with the location of the system zeros. In concluding, it is necessary to emphasize that the ultimate accuracy can, of course, never be achieved since this would involve infinite feedback gains and infinite amplitudes. The results of this paper, however, give an idea of the ideal performance of which the system is capable. In practice, these limits sometimes may not be closely approximated because of the constraints on the input amplitudes, or the presence of measurement noise.

\section{REFERENCES}

[1] I. M. Horowitz, Synthesis of Feedback Systems. New York: Academic, 1963.

[2] M. Athans and P. L. Falb, Optimal Control: An Introduction to the Theory and its Applications. New York: McGraw-Hill, 1966.

[3] B. Friedland, "Limiting forms of optimum stochastic linear regulators," in 1970 Joint Automatic Control Conf., Preprints, pp. $212-220$.

[4] H. Kwakernaak, "Optimal low-sensitivity linear feedback systems," A utomatica, vol. 5, no. 3, pp. 279-286, 1969.

[5] H. Kwakernaak and R. Sivan, Linear Optimal Control Systems. New York: Wiley, 1972.

[6] P. H. Haley, "Design of low order feedback controllers for linear multivariable systems," Dep. Eng.-Econ. Syst., Stanford Univ., Stanford, Calif., Rep. CCS-10, May 1967.

[7] W. H. Wonham, "On a matrix Riccati equation of stochastic control," SIAM J. Contr., vol. 6, no. 4, pp. 681-697, 1968.

[8] R. Sivan, "On zeroing the output and maintaining it zero," IEEE Trans. Automat. Contr. (Short Papers), vol. AC-10, pp. 193-194, Apr. 1965.

[9] S. Levy and R. Sivan, "On the stability of a zero-output system," IEEE Trans. Automat. Contr. (Corresp.), vol. AC-11, pp. 315-316, Apr. 1966.

[10] A. E. Bryson and Y. C. Ho, Applied Optimal Control. Waltham, Mass.: Blaisdeil, 1969 , pp. 246-251.

[11] H. Kwakernaak and R. Sivan, "Asymptotic pole locations of 
time-invariant linear optimal regulators," in Proc. Brd Annu. Southeast. Symp. System Theory, 1971.

[12] R. E. Kalman, "When is a linear control system optimal?" Trans. ASME, J. Basic Eng., ser. D, vol. 86, pp. 51-60, 1964.

[13] R. E. Kalman and R. S. Bucy, "New results in linear filtering and prediction theory," Trans. ASME, J. Basic Eng., ser. D, vol. 83, pp. $95-100,1961$.

[14] A. Ė. Bryson, Jr., and D. E. Johansen, "Linear filtering for time-varying systems using measurements containing colored noise," IEEE Trans. Automat. Contr, vol. AC-10, pp. 4-10, Jan. 1965.

[15] S. Butman, "A method for optimizing control-free costs in systems with linear controllers," IEEE Trans. Automat. Contr. (Short Papers), vol. AC-13, pp. 554-556, Oct. 1968.

[16] E. G. Gilbert, "The decoupling of multivariable systems by state feedback," SIAM J. Contr., vol. 7, Feb. 1969.

[17] B. Moore and L. Silverman, private communication, Aug. 1971.

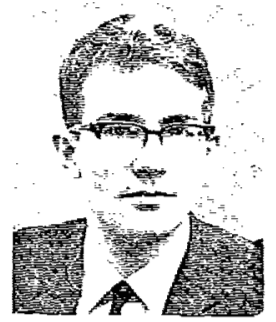

Huibert Kwakernaak (S'61-M'63) was born in Rijswijk, The Netherlands, on March 18, 1937. He completed his studies in engineering physics at the Delft University of Technology, Delft, The Netherlands, in 1960 . He received the M.S. and Ph.D. degrees from the University of California, Berkeley, in 1962 and 1963 , respectively.

From 1961 to 1963 , while at the University of California, Berkeley, he was a Research Assistant. Then in 1964 he returned to the
Delft University of Technology, where he taught and did research in the Departments of Applied Physics and Mathematics and in 1967 was appointed Lecturer. Since 1970 he has been a Professor of Applied Mathematics at the Twente University of Technology. His research interests lie in the areas of linear control theory and stochastic control and filtering theory.

Dr. Kwakernaak is a member of a number of scientific associations and is Vice Chairman of the Education Committee of the International Federation of Automatic Control and Associate Editor of Automatica.

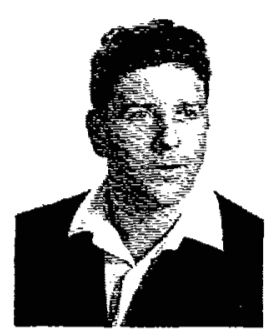

Raphael Sivan was born in Essen, Germany, on November 5, 1935. He received the B.S. degree from the Technion-Israel Institute of Technology, Haifa, in 1957 and the M.S. and Ph.D. degrees from the University of California, Berkeley, in 1961 and 1964, respectively, all in electrical engineering.

During 1963 and 1964 he was an Assistant Professor in the Department of Electrical Engineering, California Institute of Technology, Pasadena. Since then he has been on the faculty of the Technion--Israel Institute of Technology, where he is presently an Associate Professor. During 1970 and 1971 he spent his sabbatical year as a National Academy of Science Senior Research Fellow at NASA Langley Research Center, Hampton, Va.

Dr. Sivan is a member of Sigma Xi.

\title{
Instability of Slowly Varying Systems
}

\author{
RONALD A. SKOOG, MEMBER, IEEE, AND CLIFFORD G. Y. LAU, STUDENT MAMMBER, IEEE
}

\begin{abstract}
Instability criteria are obtained for systems described by $\dot{x}=A(t) \dot{x}$ when the parameters are slowly varying. In particular it is shown that, when $A(t)$ has eigenvalues in the right-half plane and all eigenvalues are bounded away from the imaginary axis, then if $\sup _{t} \geq 0\|\dot{A}(t)\|$ is sufficiently small, the system has unbounded solutions. Results are also given for systems of the form $\dot{x}=A(t) x$ $+f(x, t)$, and the dichotomy of solutions is studied in both the linear and nonlinear cases.
\end{abstract}

\section{INTRODUCTION}

$\mathbf{I}$ this paper the question of instability is considered for systems described by $\dot{x}=A(t) x$ in which the parameters are "slowly varying." In particular, it is our aim to obtain conditions under which the stability properties of the time-varying system can be predicted from

Manuscript received April 14, 1971; revised September 3, 1971. Paper recommended by J. C. Willems, Chairman of the IEEE S-CS Stability, Nonlinear Systems, Distributed Systems Committee. This research was sponsored by the Joint Services Electronies Program, Grant AFOSR-68-1488, and the National Science Foundation, Grant GK-5786.

R. A. Skoog was with the Department of Electrical Engineering and Computer Sciences and the Flectronics Research Laboratory, University of California, Berkeley, Calif. He is now with Bell Telephone Laboratories, Inc., Holmdel, N.J.

C. G. Y. Lau is with the Naval Missile Center, Point M.Tugu, Calif. the stability properties of the frozen-time systems [i.e., from the eigenvalues of $A(t)]$. Regarding stability, it is known that, if the eigenvalues of $A(t)$ lie in $\operatorname{Re} \lambda<\sigma_{0}<0$ for all $t$ and $\sup _{t \geq 0}\|\dot{A}(t)\|$ is sufficiently small, then all solutions of $\dot{x}=A(t) x$ go to zero as $t \rightarrow \infty$ (c.f., [1]-[3]). One would intuitively expect also that, if $A(t)$ had eigenvalues in the right-half plane, then the system would have unbounded solutions if $\sup _{t \geq 0}\|\dot{A}(t)\|$ was sufficiently small. It is shown here that this is indeed the case provided that no eigenvalues cross the imaginary axis. It is also shown by an example (Section III) that, if the eigenvalues are allowed to cross the imaginary axis, then even though there is always an eigenvalue with positive real part, the system can be asymptotically stable for arbitrarily $\operatorname{small} \sup _{t \geq 0}\|\dot{A}(t)\|$. Thus, this additional restriction is unavoidable for the preceding type of result to hold. These results are also extended in a straightforward manner to nonlinear systems of the form $\dot{x}=A(t) x+$ $f(x, t)$ where $\|f(x, t)\| /\|x\| \rightarrow 0$ as $\|x\| \rightarrow 0$.

The main result is proved along lines similar to the proof of the stability criteria of [1]-[3] in which Lyapunov methods were used. However, the method of constructing a Lyapunov function used in [1]-[3] cannot be used in the 